\title{
OTIMIZAÇÃO DOS ATERROS SANITÁRIOS *
}

\author{
Luciana Gandelini $^{* *}$
}

\section{José Vicente Caixeta Filho ${ }^{* * *}$}

RESUMO A escolha do local para acomodar resíduos sólidos deve obedecer a várias normas de caráter ambiental, operacional e econômico. No Estado de São Paulo, a análise de uma unidade receptora passa necessariamente pela avaliação da Cetesb - Companhia de Tecnologia de Saneamento Ambiental, que tem sinalizado sobre a qualidade e a eficiência dos aterros paulistas através da formulação do IQR (Índice de Qualidade do Aterro). O presente trabalho, com o auxílio de modelos matemáticos de otimização, mais especificamente através da linguagem de otimização GAMS, visa a avaliar os melhores locais para aterros sanitários e os melhores fluxos de resíduos entre algumas cidades do Estado de São Paulo. A partir dos resultados obtidos, infere-se que, se houvesse a conscientização plena ao se aterrar os resíduos, o uso de locais se restringiria àqueles que apresentassem melhores IQRs, acarretando maiores gastos, pois nem todo município possuiria áreas em condições ambientais adequadas para destinar seus restos, tendo de deslocá-los para outros lugares, normalmente muito distantes.

Palavras-chave: meio ambiente; pesquisa operacional; bacias hidrográficas

Código JEL: R12 - Size and spatial distributions of regional economic activity; interregional trade; Q5 - Environmental economics

* Artigo recebido em 13 de setembro de 2006 e aprovado em 16 de abril de 2007. Artigo baseado na monografia da primeira autora sob orientação do segundo autor. Os autores agradecem à Fundação de Amparo à Pesquisa do Estado de São Paulo - Fapesp o apoio dado a este trabalho.

** Economista pela Escola Superior de Agricultura Luiz de Queiroz (ESALQ) da Universidade de São Paulo (USP), e-mail: lgandeli@terra.com.br

*** (Autor-correspondente) Engenheiro civil, professor titular do Departamento de Economia, Administração e Sociologia da ESALQ/USP, e-mail: jvcaixet@esalq.usp.br 


\section{OPTIMIZATION OF THE LOCATION FOR LANDFILLS AND NOXIOUS FACILITIES IN SÃO PAULO STATE}

ABSTRACT The site selection to place solid wastes is subject to several environmental, operational and economical constraints. In São Paulo State, a possible reception unit is usually evaluated by the environmental company Cetesb, which has remarked on the quality and efficiency of a landfill through a quality index namely IQR. This paper, with the help of optimization mathematical models coded in GAMS language, seeks to evaluate the best places for the sanitary landfills and the best flow of wastes between selected cities in São Paulo State. From the results obtained, the optimal solution was regarding sites with best IQRs, but implying higher expenses because not all the cities would have areas with adequate environmental conditions to put their wastes, making them to send the wastes to other remote places.

Key words: environment; operations research; water basins 


\section{INTRODUÇÃO}

As revoluções tecnológicas, as mudanças de hábitos e de costumes das populações e o desenvolvimento desenfreado da economia em diversas regiões do mundo fazem com que o meio ambiente sofra conseqüências drásticas e muitas vezes difíceis de serem reparadas. Um dos muitos problemas que surgiram em decorrência de tal contexto refere-se ao problema dos resíduos sólidos urbanos, mais comumente conhecidos por lixo.

Conforme a população mundial cresce, há a necessidade de se produzir mais alimentos e outros bens de consumo direto. Assim, é indispensável a instalação de novas fábricas e indústrias para atender a essa demanda crescente. Conseqüentemente, eleva-se a produção de resíduos sólidos que comprometem o meio ambiente se armazenados de forma inadequada.

De acordo com Cunha (2001), a quantidade de lixo produzida atualmente no mundo tem sido grande, e o seu mau gerenciamento, além de provocar gastos financeiros significativos e sérios danos ao meio ambiente, pode comprometer a saúde e o bem-estar da população.

É evidente que as produções de resíduos sólidos não são homogêneas em todas as partes do mundo. Em regiões onde a renda per capita é mais elevada, a produção de lixo é relativamente maior. Cada país possui suas particularidades em relação à produção de resíduos, e, mais especificamente, cada município pode observar situações bastante diferenciadas.

Considerando-se o Brasil, é inegável que o Estado de São Paulo tem sido o destaque nacional em termos de crescimento industrial e urbano. Dessa forma, a produção de lixo na região cresceu com esse desenvolvimento, assim como aumentou também o número de unidades receptoras desses materiais.

O presente trabalho tem por objetivo, através da linguagem de otimização GAMS ${ }^{1}$ (Brooke et al., 1992), propor uma aplicação de modelos matemáticos visando a localizar, em um contexto intermunicipal, os melhores locais para aterros sanitários e os melhores fluxos de resíduos entre as cidades localizadas em região específica do Estado de São Paulo. 


\section{1. ÁREA dE ESTUdo}

O “Inventário Estadual de Resíduos Sólidos” do ano 2000, formulado pela Companhia de Tecnologia de Saneamento Ambiental (Cetesb), apresenta o Estado de São Paulo subdividido em bacias hidrográficas, segundo a classificação da Secretaria Estadual de Recursos Hídricos, Saneamento e Obras, que acabam por caracterizar as chamadas UGRHIs (Unidades de Gerenciamento de Recursos Hídricos).

O Estado de São Paulo possui 22 UGRHIs (figura 1), estando contidos nelas os 645 municípios paulistas. Cada qual possui suas particularidades em termos de desenvolvimento econômico, população e, conseqüentemente, produção de lixo.

Pretendendo-se analisar uma região específica, optou-se pela UGRHI 5 (associada às bacias dos rios Piracicaba, Jundiaí e Capivari), que é formada por 57 cidades e produz cerca de 2.196,7 toneladas de lixo por dia. Tal decisão foi facilitada pelo fato de este trabalho estar sendo realizado na área de abrangência dessa UGRHI, mais especificamente no Município de Piracicaba. A figura 2 destaca a localização da UGRHI 5 no Estado de São Paulo.

Na tabela 1 são apresentadas as cidades que compõem a UGRHI 5, consideradas na formulação do modelo, assim como as quantidades de resíduos produzidas em cada uma delas e as capacidades disponíveis de seus aterros sanitários ou lixões.

Nota-se que 11 municípios da UGRHI 5 possuem capacidades de seus aterros iguais a zero, implicando que os mesmos depositam seus resíduos fora de seus limites territoriais.

\section{MATERIAIS E MÉTODOS}

\subsection{Obtenção das informações}

Para a elaboração do trabalho, inicialmente foi realizado um levantamento dos depósitos de resíduos sólidos urbanos existentes na UGRHI 5 (considerando os principais aterros sanitários e lixões). Esse levantamento baseou-se no "Inventário Estadual de Resíduos Sólidos" formulado pela Cetesb (2000).

Informações referentes às áreas ocupadas pelas unidades receptoras, população e quantidade de lixo produzida por cada cidade estudada também foram retiradas do Inventário produzido pela Cetesb (2000). 


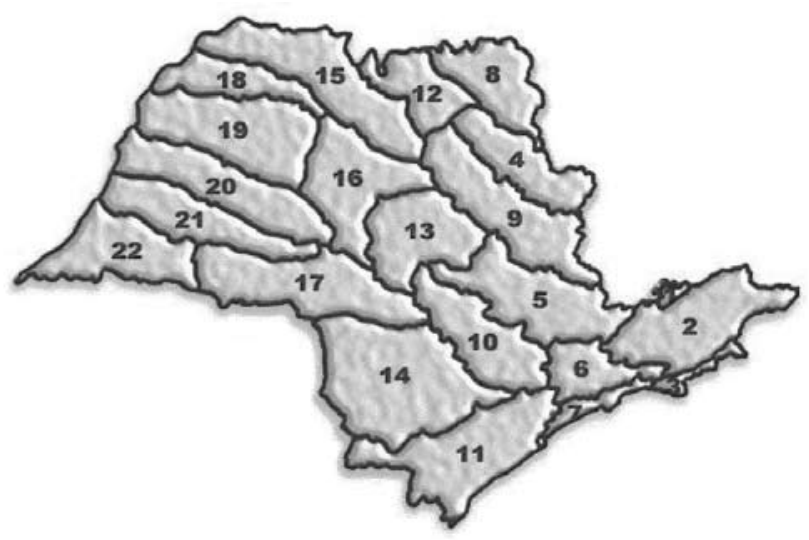

Figura 2: Localização da UGRHI 5 no Estado de São Paulo

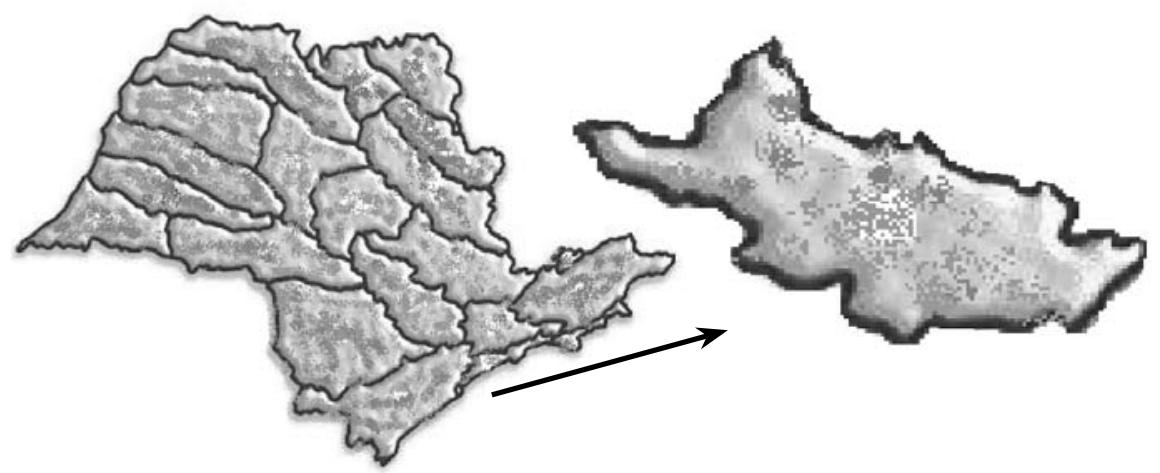


Tabela 1: Quantidade de lixo produzida diariamente e capacidade dos aterros dos municípios da UGRHI 5 que compõem o modelo

\begin{tabular}{|c|c|c|}
\hline Municípios & Quantidade de lixo/dia (t) & Capacidade dos aterros $\left(\mathrm{m}^{2}\right)$ \\
\hline Elias Fausto & 4,10 & $17.500,00$ \\
\hline Santo Antônio de Posse & 5,90 & $13.000,00$ \\
\hline Atibaia & 38,70 & $65.000,00$ \\
\hline Bom Jesus dos Perdões & 4,50 & $10.000,00$ \\
\hline Cosmópolis & 17,00 & $15.000,00$ \\
\hline Rafard & 2,90 & $30.000,00$ \\
\hline Capivari & 13,40 & $9.000,00$ \\
\hline Artur Nogueira & 12,20 & $5.000,00$ \\
\hline Cordeirópolis & 6,40 & $7.000,00$ \\
\hline Piracaia & 9,20 & $9.680,00$ \\
\hline Joanópolis & 4,20 & $3.000,00$ \\
\hline Pinhalzinho & 2,10 & $10.000,00$ \\
\hline Águas de São Pedro & 0,70 & 0 \\
\hline São Pedro & 9,00 & $24.000,00$ \\
\hline Nazaré Paulista & 2,30 & $3.000,00$ \\
\hline Americana & 90,80 & $150.125,00$ \\
\hline Hortolândia & 75,80 & $50.123,00$ \\
\hline Sumaré & 96,60 & 0 \\
\hline Pedra Bela & 0,50 & $1.227,80$ \\
\hline Tuiuti & 0,90 & $24.037,00$ \\
\hline Iracemápolis & 5,90 & $2.000,00$ \\
\hline Analândia & 1,10 & $26.494,50$ \\
\hline Vargem & 1,00 & $1.080,00$ \\
\hline Pedreira & 13,70 & $38.393,00$ \\
\hline Campinas & 666,30 & $41.300,00$ \\
\hline Limeira & 142,80 & $40.000,00$ \\
\hline Monte Mor & 13,60 & $24.200,00$ \\
\hline Santa Bárbara d'Oeste & 83,80 & $80.000,00$ \\
\hline Ipeúna & 1,40 & $12.600,00$ \\
\hline
\end{tabular}

Para a avaliação da localização dos aterros e lixões foi necessário o cálculo das distâncias rodoviárias entre as cidades que compõem o modelo. Isso se fez necessário porque as distâncias entre os municípios são fatores determinantes para se apontar quais deles serão receptores de quais unidades geradoras de resíduos.

As distâncias rodoviárias foram obtidas através de um serviço disponibilizado no site www.portaldaviagem.com.br, que, a partir das informações referentes às cidades de origem e de destino, fornece automaticamente a respectiva quilometragem. 
Continuação da tabela 1

\begin{tabular}{|c|c|c|}
\hline Municípios & Quantidade de lixo/dia (t) & Capacidade dos aterros $\left(\mathrm{m}^{2}\right)$ \\
\hline Morungaba & 3,10 & $25.000,00$ \\
\hline Santa Maria da Serra & 1,60 & $39.000,00$ \\
\hline Itupeva & 7,70 & $4.000,00$ \\
\hline Mombuca & 0,90 & 0 \\
\hline Piracicaba & 189,90 & $148.000,00$ \\
\hline Rio das Pedras & 8,80 & $10.244,00$ \\
\hline Saltinho & 1,90 & 0 \\
\hline Rio Claro & 81,70 & $43.758,50$ \\
\hline Santa Gertrudes & 6,20 & 0 \\
\hline Corumbataí & 0,70 & $24.211,30$ \\
\hline Valinhos & 31,30 & $193.367,40$ \\
\hline Amparo & 17,30 & $51.715,00$ \\
\hline Itatiba & 26,20 & $173.863,00$ \\
\hline Monte Alegre do Sul & 1,30 & 0 \\
\hline Campo Limpo Paulista & 24,90 & 0 \\
\hline Indaiatuba & 72,30 & $40.000,00$ \\
\hline Jundiaí & 179,80 & 0 \\
\hline Louveira & 8,80 & 0 \\
\hline Várzea Paulista & 37,10 & $128.000,00$ \\
\hline Vinhedo & 18,40 & 0 \\
\hline Paulínea & 20,30 & $60.800,00$ \\
\hline Salto & 36,80 & $60.000,00$ \\
\hline Jarinu & 4,60 & $59.000,00$ \\
\hline Bragança Paulista & 55,50 & $48.575,00$ \\
\hline Charqueada & 4,70 & $24.199,50$ \\
\hline Holambra & 1,60 & $30.000,00$ \\
\hline Nova Odessa & 16,40 & $26.682,30$ \\
\hline Jaguariúna & 10,30 & 0 \\
\hline
\end{tabular}

Fonte: Elaboração própria a partir de Cetesb (2000).

Para a formulação do modelo foi necessária a caracterização dos locais receptores de resíduos, sendo para isso utilizado o IQR (Índice de Qualidade de Aterro de Resíduos).

O cálculo do IQR é feito com base em levantamento realizado por técnicos da Cetesb, sobre as características locacionais, de infra-estrutura e operacionais dos sistemas de tratamento de lixo, considerando a população urbana de cada cidade e a produção de resíduos per capita. O levantamento dos dados pertinentes através da aplicação de formulários padronizados, que vem sendo realizado desde 1997, considera 41 variáveis, às quais são 
atribuídas notas específicas. A partir da contabilização da média dessas notas, tem-se o IQR de cada instalação receptora de resíduos em operação no Estado de São Paulo, que pode variar de 0 a 10, de tal forma que os aterros são classificados como inadequados, controlados e adequados (tabela 2). No caso da UGRHI 5, os IQRs respectivos são apresentados na tabela 3.

Este estudo considera ainda somente dois tipos de custos em seu modelo matemático: os custos operacionais e o custo de transporte do lixo. Lembrando que modelos são simplificações da realidade, não foram incorporados os custos fixos e os custos de preparação das áreas para serem receptoras dos resíduos.

Além disso, os valores de custos adotados para todos os municípios selecionados para este trabalho se referem aos praticados no Município de Piracicaba. Certamente esses valores não são necessariamente iguais para as demais localidades; entretanto, aplica-se tal valor devido à complexidade de se obter informações mais precisas de outros municípios. ${ }^{2}$

\subsection{Os custos operacionais}

É importante, para a obtenção dos resultados, a inclusão nos modelos dos custos operacionais dos aterros, ou seja, dos gastos realizados com as manutenções e reparos que os aterros demandam, juntamente com os dispêndios necessários para acomodar os resíduos no solo. Assim, esses custos foram calculados a partir de um valor médio de $\mathrm{R} \$ 15,00 / \mathrm{t}$ por dia de lixo aterrado para cada aterro, valor esse praticado na cidade de Piracicaba.

Dessa forma, para cada município selecionado, é obtido o produto entre a quantidade de lixo produzido por dia e a média do custo de manutenção e disposição.

Tabela 2: Enquadramentos das instalações de destinação final de lixo em função dos valores de IQR

\begin{tabular}{ll}
\hline $\mathrm{IQR} / \mathrm{IQC}$ & Enquadramento \\
\hline $0,0 \leq \mathrm{IQR} \leq 6,0$ & Condições Inadequadas \\
\hline $6,0<\mathrm{IQR} \leq 8,0$ & Condições Controladas \\
\hline $8,0<\mathrm{IQR} \leq 10,0$ & Condições Adequadas \\
\hline Fonte: Cetesb, 2000. &
\end{tabular}


Tabela 3: Municípios da UGRHI 5 e seus IQRS

\begin{tabular}{|c|c|c|c|}
\hline Municípios - UGRHI 5 & IQR & Municípios - UGRHI 5 & IQR \\
\hline Elias Fausto & 1,8 & Morungaba & 0 \\
\hline Santo Antônio de Posse & 1,9 & Santa Maria da Serra & 0 \\
\hline Atibaia & 2,2 & Itupeva & 7,7 \\
\hline Bom Jesus dos Perdões & 2,8 & Mombuca & 0 \\
\hline Cosmópolis & 2,8 & Piracicaba & 7,8 \\
\hline$\underline{\text { Rafard }}$ & 2,8 & Rio das Pedras & 7,8 \\
\hline Capivari & 3 & Saltinho & 0 \\
\hline Artur Nogueira & 3,3 & Rio Claro & 7,9 \\
\hline Cordeirópolis & 3,3 & Santa Gertrudes & 7,9 \\
\hline Piracaia & 3,4 & Corumbataí & 8,2 \\
\hline Joanópolis & 3,5 & Valinhos & 8,3 \\
\hline Pinhalzinho & 3,9 & Amparo & 8,5 \\
\hline Águas de São Pedro & 0 & Itatiba & 8,5 \\
\hline São Pedro & 4,3 & Monte Alegre do Sul & 0 \\
\hline Nazaré Paulista & 4,5 & Campo Limpo Paulista & 0 \\
\hline Americana & 4,8 & Indaiatuba & 8,7 \\
\hline Hortolândia & 5,1 & Jundiaí & 0 \\
\hline Sumaré & 0 & Louveira & 0 \\
\hline Pedra Bela & 0 & Várzea Paulista & 8,7 \\
\hline Tuiuti & 5,5 & Vinhedo & 0 \\
\hline Iracemápolis & 5,6 & Paulínea & 8,9 \\
\hline Analândia & 6,3 & Salto & 8,9 \\
\hline Vargem & 6,3 & Jarinu & 9 \\
\hline Pedreira & 6,4 & Bragança Paulista & 9,2 \\
\hline Campinas & 6,5 & Charqueada & 9,2 \\
\hline Limeira & 6,8 & Holambra & 9,3 \\
\hline Monte Mor & 0 & Nova Odessa & 9,3 \\
\hline Santa Bárbara d'Oeste & 7,1 & Jaguariúna & 0 \\
\hline Ipeúna & 0 & & \\
\hline
\end{tabular}

\subsection{Custo do transporte do lixo}

$\mathrm{O}$ custo do transporte do lixo também é incorporado ao modelo. $\mathrm{O}$ valor usado como referência é um valor médio do transporte de uma tonelada de resíduos por quilômetro obtido através de dados coletados por Cunha (2001) para o Município de Piracicaba.

Em seu trabalho, Cunha (2001) obteve dados de coleta de resíduos para 27 setores atendidos na cidade de Piracicaba. Tais dados fornecem, por mês, 
as distâncias percorridas nesses setores até a unidade receptora, as quantidades de lixo movimentadas em cada um dos setores e o custo por quilômetro para transportar essas quantidades. Assim, através do processamento desses dados, obteve-se que o custo médio para o frete é de $\mathrm{R} \$ 0,0174 / \mathrm{t} . \mathrm{km}$.

\subsection{Representação do modelo matemático}

O modelo adotado neste estudo envolve uma estrutura de programação inteira mista e refere-se à minimização de uma função objetiva. Nela, estão incorporados os custos considerados para a obtenção da localização ótima das unidades receptoras de resíduos (custo de transporte dos resíduos e custo operacional dos aterros), compreendendo $m$ localidades produtoras e $n$ receptoras de lixo, e estando sujeita a várias restrições de ordem física e comportamental. A seguir, apresentam-se as especificações das equações e inequações do modelo básico proposto.

Função objetivo:

$$
\operatorname{Min} Z=\sum_{i=1}^{m} \sum_{j=1}^{n} \mathrm{~A}_{i j} x_{i j} C_{i j}+\sum_{j=1}^{n} F_{j} C O_{j}
$$

em que:

$Z=$ valor da função objetivo.

$\mathrm{A}_{i j}=$ distância rodoviária do município $i$ ao município $j$.

$x_{i j}=$ quantidade de lixo produzido no município $i$ e aterrado no município $j$.

$C_{i j}=$ custo de transporte do lixo produzido no município $i$ e aterrado no município $j$.

$F_{j}=$ variável binária, tipo zero-um, associada à recepção ou não de lixo por um aterro sanitário (ou lixão) localizado no município $j$.

$C \mathrm{O}_{j}=$ custo operacional (manutenção e disposição) do aterro ou lixão localizado no município $j$.

Restrições:

a) A movimentação de lixo a partir do município $i$ não deve exceder a produção do próprio município.

$$
\sum_{j=1}^{n} x_{i j}-S_{i} \leq 0, \text { para todo } i
$$


em que:

$\sum_{j=1}^{n} x_{i j}=$ quantidade total de lixo produzido no município $i$ e transportada para os municípios $j$.

$S_{i}=$ quantidade de lixo produzido no município $i$.

b) Capacidade de recepção dos aterros sanitários e/ou lixões.

$$
\sum_{i=1}^{m} x_{i j} \leq D_{j} F_{j}, \text { para todo } j
$$

em que:

$\sum_{i=1}^{m} x_{i j}=$ quantidade de lixo recebida dos municípios produtores de lixo no aterro do município $j$.

$D_{j}=$ capacidade total de recepção de resíduos no município $j$.

c) Existência de pelo menos uma unidade receptora de resíduos no Estado de São Paulo.

$$
\sum_{i=1}^{n} F_{j} \geq 1
$$

d) Exclusão dos aterros considerados inadequados, ou seja, cuja média de seus IQRs sejam inferiores a $k$.

$x_{i j}=0 \quad$ se, $P_{j} \leq k$ para todo $i$ e $j$.

em que:

$P_{j}=$ valor do IQR do município $j$.

$k=$ IQR mínimo exigido para que um município possa ser selecionado.

$x_{i j}=$ quantidade de lixo produzido no município $i$ e aterrado no município $j$.

\subsection{0 modelo e seus cenários}

O modelo envolve os 57 municípios $(m=n=57)$ que compõem a UGRHI 5. Os três cenários que o compõem estão especificados a seguir. 
(1) O cenário 1: O modelo com $k=8,1$ na equação (5) representa o que se chamou de cenário 1 . Nele, especificou-se que deveria haver pelo menos um aterro sanitário ou lixão selecionado para a recepção dos resíduos em toda a UGRHI 5, não existindo então um limite de unidades receptoras, ou seja, todas as disponíveis poderiam ser utilizadas. Além disso, determinou-se que somente os municípios com IQRs iguais ou superiores a 8,1 , ou seja, aqueles adequados, poderiam ser selecionados.

(2) O cenário 2: Um segundo cenário formulado foi o denominado cenário 2, em que se buscou uma outra alternativa para a obtenção dos resultados. Nesse cenário, determinou-se assim que os aterros controlados poderiam fazer parte do resultado, ou seja, as unidades com IQRs maiores ou iguais a $6,1(k=6,1$ na equação $(5))$ poderiam fazer parte da solução encontrada.

(3) O cenário 3: Foi proposto também um terceiro cenário para o modelo, que foi denominado cenário 3. Nesse cenário não foram consideradas as variáveis de caracterização dos aterros, ou seja, a restrição envolvendo o IQR, representada pela equação (5), não foi considerada.

\section{RESULTADOS 3 E DISCUSSÃO}

\subsection{0 cenário 1}

Nesse primeiro cenário foi obtido o valor de $\mathrm{R} \$ 1.501,81$, referente à minimização dos custos. Quatro municípios foram selecionados para serem receptores de resíduos. A cidade de Nova Odessa, segundo os resultados obtidos, acomodaria 71,97\% do total produzido, ou seja, receberia a maioria das 2.196,90 toneladas produzidas diariamente pelos 57 municípios da UGRHI 5 que compõem o modelo. As cidades de Jarinu, Corumbataí e Holambra acomodariam, respectivamente, $19,51,4,88$ e 3,65\% do montante gerado.

\subsection{0 cenário 2}

A solução referente à minimização dos custos considerados para a obtenção da localização ótima das unidades receptoras de resíduos equivale a $\mathrm{R} \$$ $1.471,46$ (2,02\% inferior ao cenário anterior). 
Foram selecionadas cinco cidades para serem receptoras de resíduos nesse cenário. A cidade de Nova Odessa ficou responsável pelo aterramento de $65,57 \%$ do total de 2.196,90 toneladas produzidas diariamente pelos municípios da UGRHI 5. Os Municípios de Itupeva, Vargem, Corumbataí e Holambra receberiam, respectivamente, $20,53,5,41,4,88$ e 3,61\% do restante a ser acomodado.

\subsection{0 cenário 3}

No cenário 3, em que não se consideram as variáveis de caracterização dos aterros (IQRs), verificou-se que os custos considerados para a obtenção da localização ótima das unidades receptoras de resíduos equivalem a $\mathrm{R} \$ 1.422,13$ (3,35\% inferior ao cenário 2 e 5,30\% inferior ao cenário 1).

Cinco municípios foram selecionados para serem depósitos de resíduos. A cidade de Nova Odessa ficou responsável pelo aterramento de 51,25\% do total produzido, recebendo a maioria das $2.196,90$ toneladas produzidas diariamente pelos 57 municípios que compõem o modelo. As cidades de Iracemápolis e Itupeva alojariam, respectivamente, 21,07 e 20,39\% do montante produzido. Os depósitos localizados em Vargem e Tuiuti se incumbiriam de aterrar 7,29\% do lixo restante.

\section{CONCLUSÕES}

Através dos resultados obtidos nos três cenários apresentados neste trabalho, pôde-se comprovar, com a minimização dos custos considerados para a obtenção da localização ótima das unidades receptoras de resíduos, que, ao se considerar os IQRs dos aterros - fator limitante para a existência de locais receptores - as despesas aumentam.

$\mathrm{Na}$ tabela 4, percebe-se que, conforme se limitam, em termos de IQR, as localidades que devem ser utilizadas como depósitos de lixo, os custos de disposição de materiais se elevam.

A não-imposição da seleção de aterros adequados no modelo implica reduções de custos. Assim, a diferença dos custos diários no cenário que limita as unidades receptoras às adequadas (cenário 1) e no que permite a seleção de qualquer local segundo seu IQR (cenário 3) chegou a 5,30\%. Portanto, seria mais econômico depositar resíduos em locais considerados, se- 
Tabela 4: Custos resultantes através da localização ótima das unidades receptoras de resíduos, por tipo de cenário considerado

\begin{tabular}{cc}
\hline & Modelo "micro" \\
\hline Cenário & Custo \\
\hline $1(\mathrm{IQR} \geq 8,1)$ & $\mathrm{R} \$ 1.501,81$ \\
\hline $2(\mathrm{IQR} \geq 6,1)$ & $\mathrm{R} \$ 1.471,46$ \\
\hline $3($ sem IQR) & $\mathrm{R} \$ 1.422,13$ \\
\hline Fonte: Resultados da pesquisa. &
\end{tabular}

gundo a classificação da Cetesb, inadequados. Entretanto, os danos ambientais causados no local seriam imensos e, caso houvesse interesse futuro em restaurar a área, as despesas seriam demasiadamente elevadas, considerando-se que nada foi desenvolvido ao longo da existência do aterro para se poupar a área. ${ }^{4}$

Através dos resultados obtidos neste trabalho, aponta-se que, tecnicamente, é possível o recebimento de resíduos de vários municípios por poucas unidades receptoras. Entretanto, o que se observa na prática é uma certa tendência de se aterrar os resíduos nas mesmas localidades onde são produzidos.

Contudo, a maior intenção deste trabalho está em apontar que a formação de lixo e, conseqüentemente, a sua disposição são fatos que necessitam ser constantemente analisados, já que os custos envolvidos em um processo de aterramento considerado adequado podem ser bem mais altos que a execução de um tipo de disposição a esmo. Além disso, existe outro ponto importante em termos do local e da forma de se destinar resíduos: a saúde de seres humanos e da natureza, pois, nesse caso, não está em jogo somente a qualidade de vida dos habitantes do tempo presente, mas também a qualidade de vida das gerações futuras.

O "Inventário Estadual de Resíduos Sólidos" continua sendo atualizado pela Cetesb. De acordo com Margarido (2003), a avaliação dos locais receptores de lixo do Estado de São Paulo para o ano 2002 revela que ocorreram significativas mudanças em algumas das cidades analisadas neste estudo.

Portanto, dado que as características dos locais receptores de resíduos alteram-se significativamente ao longo do tempo, recomenda-se aos órgãos fiscalizadores e ao Poder Público que utilizem ferramentas adequadas, tal como o modelo proposto neste estudo, para facilitar o monitoramento e controle da utilização de lixões e aterros sanitários. 


\section{NOTAS}

1. Para o processamento do modelo de programação mista (variáveis contínuas e discretas) proposto neste artigo, através da linguagem de otimização GAMS, foi utilizado o método numérico (solver) CPLEX, que para problemas dessa natureza faz o uso de código computacional baseado no algoritmo branch-and-bound e com tolerância tal que garante que a solução (em existindo) a ser obtida represente efetivamente o ponto de ótimo global.

2. Durante a execução do trabalho, foram realizados contatos com as prefeituras de cada um dos municípios envolvidos e constatou-se que esses dados são de difícil acesso. Mesmo sendo o próprio órgão público ou até mesmo alguma instituição privada responsável pela coleta e disposição final dos resíduos, não se têm documentadas com exatidão tais informações. Recomenda-se que, em trabalhos futuros, recursos sejam alocados para a obtenção de detalhamento de tais custos.

3. As tabelas com os resultados completos obtidos para cada um dos cenários podem ser consultadas em Gandelini (2002).

4. A introdução do IQR não eleva significativamente o custo (aproximadamente 5\%) quando se comparam os resultados dos cenários 3 (sem IQR) e 1 (com IQR $\geq 8,1$ ). Porém, tal como destacado por um dos revisores (anônimos) deste artigo (a quem agradecemos), essa vem sendo efetivamente a diferença entre "o racional e o absurdo irracional".

\section{REFERÊNCIAS BIBLIOGRÁFICAS}

BROOKE, R.; KENDRICK, D.; MEERAUS, A. GAMS: A user's guide, release 2.25. The Scientific Press. 1992.289 p.

CAIXETA FILHO, J. V. Pesquisa operacional: técnicas de otimização aplicadas a sistemas agroindustriais. Piracicaba: Atlas, 2001.

CETESB. Companhia de Tecnologia de Saneamento Ambiental. Inventário estadual de resíduos sólidos domiciliares. São Paulo, 2000. (Relatório Síntese). Disponível em: www.cetesb.sp.gov.br

Companhia de Tecnologia de Saneamento Ambiental. Aterros sanitários. Apostilas Ambientais. São Paulo, 1997.

CUNHA, V. Gerenciamento da coleta de resíduos sólidos urbanos: estruturação e aplicação de modelo não-linear de programação por metas. Piracicaba, 2001. Dissertação (M. S.). Escola Superior de Agricultura Luiz de Queiroz. Universidade de São Paulo.

GANDELINI, L. Localização de aterros sanitários e lixões no Estado de São Paulo, considerando padrões ambientais distintos: uma aplicação de modelos matemáticos de otimização. Piracicaba, 2002. Monografia (B. S.). Escola Superior de Agricultura Luiz de Queiroz. Universidade de São Paulo.

MARGARIDO, A. P. Cetesb reprova 52,9\% dos lixões da região. Folha de S. Paulo, São Paulo, p. C1, 23/01/2003.

SITE: www.portal da viagem.com.br 
